\title{
The Uniqueness Problem of Sequence Product on Operator Effect Algebra $\mathcal{E}(H)^{*}$
}

\author{
Liu Weihua, Wu Junde \\ Department of Mathematics, Zhejiang University, Hangzhou 310027, P. R. China
}

\begin{abstract}
A quantum effect is an operator on a complex Hilbert space $H$ that satisfies $0 \leq A \leq I$. $W e$ denote the set of all quantum effects by $\mathcal{E}(H)$. In this paper we prove, Theorem 4.3, on the theory of sequential product on $\mathcal{E}(H)$ which shows, in fact, that there are sequential products on $\mathcal{E}(H)$ which are not of the generalized Lüders form. This result answers a Gudder's open problem negatively.
\end{abstract}

PACS numbers: 02.10-v, 02.30.Tb, 03.65.Ta.

\section{Introduction}

If a quantum-mechanical system $\mathcal{S}$ is represented in the usual way by a complex Hilbert space $H$, then a self-adjoint operator $A$ on $H$ such that $0 \leq A \leq I$ is called the quantum effect on $H([1,2])$. Quantum effects represent yes-no measurements that may be unsharp. The set of quantum effects on $H$ is denoted by $\mathcal{E}(H)$. The subset $\mathcal{P}(H)$ of $\mathcal{E}(H)$ consisting of orthogonal projections represents sharp yes-no measurements. Let $\mathcal{T}(H)$ be the set of trace class operators on $H$ and $\mathcal{S}(H)$ the set of density operators, i.e., the trace class positive operators on $H$ of unit trace, which represent the states of quantum system. An operation is a positive linear mapping $\Phi: \mathcal{T}(H) \rightarrow \mathcal{T}(H)$ such that for each $T \in \mathcal{S}(H)$, $0 \leq \operatorname{tr}[\Phi(T)] \leq 1([3-5])$. Each operation $\Phi$ can define a unique quantum effect $B$ such that for each $T \in \mathcal{T}(H), \operatorname{tr}[\Phi(T)]=\operatorname{tr}[T B]$.

Let $\mathcal{B}(H)$ be the set of bounded linear operators on $H$, the dual mapping $\Phi^{*}: \mathcal{B}(H) \rightarrow$ $\mathcal{B}(H)$ of an operation $\Phi$ is defined by the relation $\operatorname{tr}\left[T \Phi^{*}(A)\right]=\operatorname{tr}[\Phi(T) A], A \in \mathcal{B}(H), T \in$ $\mathcal{T}(H)([4])$. The effect $B$ defined by an operation $\Phi$ satisfies that $B=\Phi^{*}(I)([5])$.

For each $P \in \mathcal{P}(H)$ is associated a so-called Lüders operation $\Phi_{L}^{P}: T \rightarrow P T P$, its dual is $\left(\Phi_{L}^{P}\right)^{*}(A)=P A P$ and the corresponding quantum effect is $\left(\Phi_{L}^{P}\right)^{*}(I)=P$. These

*E-mail: wjd@zju.edu.cn 
operations arise in the context of ideal measurements. Moreover, each quantum effect $B \in \mathcal{E}(H)$ gives to a general Lüders operation $\Phi_{L}^{B}: T \rightarrow B^{\frac{1}{2}} T B^{\frac{1}{2}}$ and $B$ is recovered as $\left(\Phi_{L}^{B}\right)^{*}(I)=B$ as well.

Let $\Phi_{1}, \Phi_{2}$ be two operations. The composition $\Phi_{2} \circ \Phi_{1}$ is a new operation, called a sequential operation as it is obtained by performing first $\Phi_{1}$ and then $\Phi_{2}$. In general, $\Phi_{2} \circ \Phi_{1} \neq \Phi_{1} \circ \Phi_{2}$. Note that for any two quantum effects $B, C \in \mathcal{E}(H)$ we have $\left(\Phi_{L}^{C} \circ \Phi_{L}^{B}\right)^{*}(I)=B^{\frac{1}{2}} C B^{\frac{1}{2}}\left(\left[5, P_{26-27}\right]\right)$. It shows that the new quantum effect $B^{\frac{1}{2}} C B^{\frac{1}{2}}$ yielded by $B$ and $C$ has important physics meaning. Professor Gudder called it the sequential product of $B$ and $C$, and denoted it by $B \circ C$. It represents the quantum effect produced by fist measuring $A$ then measuring $B([6-8])$. This sequential product has also been generalized to an algebraic structure called a sequential effect algebra ([7]).

Now, we introduce the abstract sequential product on $\mathcal{E}(H)$ as following:

Let $\circ$ be a binary operation on $\mathcal{E}(H)$, i.e., o: $\mathcal{E}(H) \times \mathcal{E}(H) \rightarrow \mathcal{E}(H)$, if it satisfies:

(S1). The map $B \rightarrow A \circ B$ is additive for each $A \in \mathcal{E}(H)$, that is, if $B+C \leq I$, then

$$
(A \circ B)+(A \circ C) \leq I \text { and }(A \circ B)+(A \circ C)=A \circ(B+C) .
$$

(S2). $I \circ A=A$ for all $A \in \mathcal{E}(H)$.

(S3). If $A \circ B=0$, then $A \circ B=B \circ A$.

(S4). If $A \circ B=B \circ A$, then $A \circ(I-B)=(I-B) \circ A$ and $A \circ(B \circ C)=(A \circ B) \circ C$ for all $C \in \mathcal{E}(H)$.

(S5). If $C \circ A=A \circ C, C \circ B=B \circ C$, then $C \circ(A \circ B)=(A \circ B) \circ C$ and

$$
C \circ(A+B)=(A+B) \circ C \text { whenever } A+B \leq I .
$$

If $\mathcal{E}(H)$ has a binary operation o satisfying conditions $(\mathrm{S} 1)-(\mathrm{S} 5)$, then $(\mathcal{E}(H), 0, I, \circ)$ is called a sequential operator effect algebra. Professor Gudder showed that for any two quantum effects $B$ and $C$, the operation o defined by $B \circ C=B^{\frac{1}{2}} C B^{\frac{1}{2}}$ satisfies conditions (S1)-(S5), and so is a sequential product of $\mathcal{E}(H)$, which we call the generalized Lüders form. In 2005, Professor Gudder presented 25 open problems about the general sequential effect algebras. The second problem is:

Problem 1.1 ([9]). Is $B \circ C=B^{\frac{1}{2}} C B^{\frac{1}{2}}$ the only sequential product on $\mathcal{E}(H)$ ?

As we see the five properties are base on the measurement logics and the the uniqueness property has been asked many times in Gudder's paper. In this paper, we construct a new sequential product on $\mathcal{E}(H)$ which differs from the generalized Lüders form, thus, we answer the open problem negatively. 


\section{Sequential Product on $\mathcal{E}(H)$}

In this section, we study some abstract properties of sequential product $\circ$ on $\mathcal{E}(H)$. For convenience, we introduce the following notations: If $A, B \in \mathcal{E}(H)$, we say that $A \oplus B$ is defined if and only if $A+B \leq I$ and define $A \oplus B=A+B$; if $A \circ B=B \circ A$, we denote $A \mid B$.

Lemma 2.1. If $A, B \in \mathcal{E}(H), a \in[0,1]$, then

$$
A \circ(a B)=a(A \circ B)
$$

Proof. It is clear that for $a=1$, the conclusion is true. If $a>0$ is a rational number, i.e., $a=\frac{m}{n}$, where $n, m$ are positive integer, it follows from $\bigoplus_{i=1}^{n}\left(A \circ \frac{1}{n} B\right)=A \circ B$ that $A \circ\left(\frac{1}{n} B\right)=$ $\frac{1}{n}(A \circ B)$, thus, $A \circ\left(\frac{m}{n} B\right)=\bigoplus_{i=1}^{m} A \circ\left(\frac{1}{n} B\right)=\frac{m}{n}(A \circ B)$. If $a \in[0,1]$ is not a rational number, then for each $q=\frac{m}{n}>a$ we have $q(A \circ B)=A \circ(q B)=A \circ[(q-a) B]+A \circ(a B) \geq A \circ(a B)$, so $q(A \circ B) \geq A \circ(a B)$. Let $q \rightarrow a$ we have $a(A \circ B) \geq A \circ(a B)$. Similarly, we can get that $A \circ(a B) \geq a(A \circ B)$ by taking $q=\frac{m}{n}<a$. So $A \circ(a B)=a(A \circ B)$. Moreover, it follows from the proof process that for $a=0$ the conclusion is also true.

Lemma 2.2 ([9], Theorem $3.4(\mathrm{i}))$. Let $A \in \mathcal{E}(H)$ and $E \in \mathcal{P}(H)$. If $A \leq E$, then $A \mid E$ and $E \circ A=A$.

Lemma 2.3. If $a \in[0,1], E \in \mathcal{P}(H)$, then $a I \mid E$ and $(a I) \circ E=E \circ(a I)=a E$.

Proof. Since $a E \leq E$, so $a E \mid E$ and $E \circ E=E$ by Lemma 2.2, it follows from $E=$ $E \circ I=(E \circ E) \oplus(E \circ(I-E))=E \oplus(E \circ(I-E))$ that $E \circ(I-E)=0$, note that $E \circ(a(I-E)) \leq E \circ(I-E)=0$, so $E \circ(a(I-E))=0$, thus, it follows from (S3) that $E \mid a(I-E)$, moreover, by (S5) we have $E \mid a(I-E) \oplus a E=a I$, so, it follows from Lemma 2.1 and Lemma 2.2 that $(a I) \circ E=E \circ(a I)=a(E \circ I)=a E$.

Lemma 2.4. If $E, F \in \mathcal{P}(H), E \leq F$ and $0 \leq a \leq 1$, then $E \mid a F$ and $E \circ(a F)=a E$.

Proof. It follows from $E \leq F$ that $I-E \geq I-F \geq a(I-F)$, by Lemma 2.2 and Lemma 2.3, we have $I-E \mid a(I-F)$ and $I-E \mid(1-a) I$, thus, $I-E \mid a(I-F) \oplus(1-a) I=I-a F$, it follows from (S4) that $E \mid I-a F$ and so by (S4) again that $E \mid a F$, moreover, by Lemma 2.1 and Lemma 2.2, we have $(a F) \circ E=E \circ(a F)=a(E \circ F)=a E$.

Lemma 2.5. If $E \in \mathcal{P}(H), A \in \mathcal{E}(H), 0 \leq a \leq 1$ and $A \leq E$, then $a E \mid A$, and $(a E) \circ A=$ $A \circ(a E)=a A$. 
Proof. It follows from Lemma 2.2 that $A \mid E$, so by (S4) we have $A \mid I-E$. Since $A \circ E=$ $A=A \circ I=A \circ E \oplus A \circ(I-E)$, so $A \circ(I-E)=0$. Note that $A \circ(a(I-E)) \leq A \circ(I-E)$, we have $A \circ(a(I-E))=0$, so $A \mid a(I-E)$.

Let $\left\{E_{\lambda}\right\}$ be the identity resolution of $A$ and denote

$$
\begin{aligned}
& A_{n}=\sum_{i=0}^{2^{n}-1} \frac{i}{2^{n}}\left(E_{\frac{i+1}{2^{n}}}-E_{\frac{i}{2^{n}}}\right), \\
& B_{n}=\sum_{i=1}^{2^{n}} \frac{i}{2^{n}}\left(E_{\frac{i}{2^{n}}}-E_{\frac{i-1}{2^{n}}}\right) .
\end{aligned}
$$

Note that $A \in \varepsilon(H)$, so $E_{\lambda}=0$ when $\lambda<0$ and $E_{\lambda}=I$ when $1 \leq \lambda$. Moreover, for each $n \in \mathbb{N}, A_{n} \leq A_{n+1}, B_{n+1} \leq B_{n}$, and when $n \rightarrow \infty,\left\|A_{n}-A\right\| \rightarrow 0,\left\|B_{n}-A\right\| \rightarrow 0$ ([10]).

Let $0 \leq b \leq 1$. Then it follows from Lemma 2.1 and Lemma 2.3 that

$$
\begin{gathered}
(b I) \circ A_{n}=\sum_{i=1}^{2^{n}-1}(b I) \circ\left(\frac{i}{2^{n}}\right)\left(E_{\frac{i+1}{2^{n}}}-E_{\frac{i}{2^{n}}}\right) \\
=\sum_{i=1}^{2^{n}-1}\left(\frac{i b}{2^{n}}\right)\left(E_{\frac{i+1}{2^{n}}}-E_{\frac{i}{2^{n}}}\right)=b A_{n}
\end{gathered}
$$

and

$$
(b I) \circ B_{n}=b B_{n}
$$

Note that $A \geq A_{n}$, so $(b I) \circ A \geq(b I) \circ A_{n}=b A_{n}$. Let $n \rightarrow \infty$. Then $(b I) \circ A \geq b A$, do the same with $\left\{B_{n}\right\}$, we get $(b I) \circ A \leq b A$, so $(b I) \circ A=b A=A \circ(b I)$. That is $A \mid b I$ for each $0 \leq b \leq 1$, in particular, $A \mid(1-a) I$. Thus, it follows from $A \mid(1-a) I+a(I-E)$ that $A \mid I-a E$, by (S4) we have $A \mid a E$, Hence, $(a E) \circ A=A \circ(a E)=a(A \circ E)=a A$.

Lemma 2.6. Let $0 \leq a \leq 1$ and $A, B \in \mathcal{E}(H)$. Then

$$
(a A) \circ B=A \circ(a B)=a(A \circ B) .
$$

Proof. It follows from Lemma 2.5 that $(a A) \circ B=(A \circ(a I)) \circ B=A \circ((a I) \circ B)=$ $A \circ(a B)=a(A \circ B)$.

Lemma 2.6 showed that we can write $a(A \circ B)$ for $(a A) \circ B$ and $A \circ(a B)$.

In order to obtain our main result in this section, we need to extent $\circ: \mathcal{E}(H) \times \mathcal{E}(H) \rightarrow$ $\mathcal{E}(H)$ to $\mathcal{E}(H) \times \mathcal{S}(H) \rightarrow \mathcal{S}(H)$, where $\mathcal{S}(H)$ is the set of bounded linear self-adjoint operators on $H$. 
Let $B \in \mathcal{E}(H), A \in \mathcal{S}^{+}(H)$. Then there exists a number $M>0$ such that $\frac{A}{M} \in \mathcal{E}(H)$. Now we define

$$
B \circ A=M\left(B \circ \frac{A}{M}\right) .
$$

If there is another positive number $M^{\prime}$ such that $\frac{A}{M^{\prime}} \in \mathcal{E}(H)$, without losing generality, we assume that $M \leq M^{\prime}$, then $M^{\prime}\left(B \circ \frac{A}{M^{\prime}}\right)=M^{\prime}\left(B \circ\left(\frac{M}{M^{\prime}} \frac{A}{M}\right)\right)=M^{\prime}\left(\frac{M}{M^{\prime}}\left(B \circ \frac{A}{M}\right)\right)=M\left(B \circ \frac{A}{M}\right)$, this showed that $B \circ A$ is well defined for each bounded linear positive operator $A$ on $H$. In general, if $A \in \mathcal{S}(H)$, we can express $A$ as $A_{1}-A_{2}$, where $A_{1}, A_{2}$ are two bounded linear positive operators on $H([10])$. Now we define

$$
B \circ A=B \circ A_{1}-B \circ A_{2}
$$

If $A_{1}^{\prime}-A_{2}^{\prime}$ is another expression of $A$ with the above properties, then $A_{1}+A_{2}^{\prime}=A_{1}^{\prime}+A_{2}=$ $K$ is a bounded linear positive operator on $H$. If take positive real number $M$ such that $\frac{K}{M} \in \mathcal{E}(H)$, then $B \circ\left(A_{1}+A_{2}^{\prime}\right)=M\left(B \circ\left(\frac{A_{1}}{M}+\frac{A_{2}^{\prime}}{M}\right)\right)=M\left(B \circ \frac{A_{1}}{M}\right)+M\left(B \circ \frac{A_{2}^{\prime}}{M}\right)=$ $B \circ A_{1}+B \circ A_{2}^{\prime}$. Similarly, $B \circ\left(A_{1}^{\prime}+A_{2}\right)=B \circ A_{1}^{\prime}+B \circ A_{2}$. Thus, it follows from $B \circ A_{1}^{\prime}+B \circ A_{2}=B \circ A_{1}+B \circ A_{2}^{\prime}, B \circ A_{1}-B \circ A_{2}=B \circ A_{1}^{\prime}-B \circ A_{2}^{\prime}$. This showed that $\circ$ is well defined on $\mathcal{E}(H) \times S(H)$.

From the above discussion we can easily prove the following important result:

Theorem 2.7. If $B \in \mathcal{E}(H), A_{1}, A_{2} \in S(H)$ and $a \in \mathbb{R}$, then we have

$$
B \circ\left(A_{1}+A_{2}\right)=B \circ A_{1}+B \circ A_{2}, B \circ\left(a A_{1}\right)=a\left(B \circ A_{1}\right) .
$$

\section{Sequential Product on $\mathcal{E}(H)$ with $\operatorname{dim}(H)=2$}

In this section, we suppose that $\operatorname{dim}(H)=2$. Now, we explore the key idea of constructing our sequential product.

Lemma 3.1. If $E \in \mathcal{P}(H), B \in \mathcal{E}(H)$, then $E \circ B=E B E$.

Proof. Since $E$ is a orthogonal projection on $\mathcal{E}(H)$ with $\operatorname{dim}(H)=2$, so there exists a normal basis $\left\{e_{1}, e_{2}\right\}$ of $H$ such that $E\left(e_{i}\right)=\lambda_{i} e_{i}$, where $\lambda_{i} \in\{0,1\}, i=1,2$. If $\lambda_{i}=0, i=1,2$, then $E=0$, if $\lambda_{i}=1, i=1,2$, then $E=I$. It is clear that for $E=0$ or $E=I$, the conclusion is true. Without losing generality, we now suppose that $\lambda_{1}=1$ and $\lambda_{2}=0$, i.e., $\left(E\left(e_{1}\right), E\left(e_{2}\right)\right)=\left(e_{1}, e_{2}\right)\left(\begin{array}{ll}1 & 0 \\ 0 & 0\end{array}\right)$. Let $B \in S(H)$. Then we have 
$\left(B\left(e_{1}\right), B\left(e_{2}\right)\right)=\left(e_{1}, e_{2}\right)\left(\begin{array}{ll}x & y \\ \bar{y} & z\end{array}\right)$, where $x, z \in \mathbb{R}([10])$. Now we define two linear operators $X$ and $Z$ on $H$ satisfy that

$$
\left(X\left(e_{1}\right), X\left(e_{2}\right)\right)=\left(e_{1}, e_{2}\right)\left(\begin{array}{ll}
x & 0 \\
0 & 0
\end{array}\right)
$$

and

$$
\left(Z\left(e_{1}\right), Z\left(e_{2}\right)\right)=\left(e_{1}, e_{2}\right)\left(\begin{array}{ll}
0 & 0 \\
0 & z
\end{array}\right) .
$$

Then $X=x E, Z=z(I-E) \in \mathcal{E}(H)$ and it follows from (S1) and Lemma 2.2 that $E \circ X=X$ and $E \circ Z=0$. Denote

$$
\left(E \circ B\left(e_{1}\right), E \circ B\left(e_{2}\right)\right)=\left(e_{1}, e_{2}\right)\left(\begin{array}{cc}
f(x, y, z) & g(x . y . z) \\
\overline{g(x . y . z)} & h(x, y, z)
\end{array}\right) .
$$

Since $S(H)$ is a real linear space and by Theorem 2.7 that $B \rightarrow E \circ B$ is a real linear map of $S(H) \rightarrow S(H)$, so $f, g$ and $h$ are real linear maps of vector $(x, y, z)$ and $f$ and $g$ are real-valued functions of $(x, y, z)$, thus, function $f(x, y, z)$ must have the form $([10])$ : $f(x, y, z)=k x+l z+n(y+\bar{y})+i m(y-\bar{y})$, where $k, l, m, n \in R$. Let $B=X$ and $B=Z$, respectively, it follows from $E \circ X=X$ and $E \circ Z=0$ that $l=0, k=1$, so $f(x, y, z)=x+n(y+\bar{y})+m i(y-\bar{y})$. Note that when $B \in \mathcal{S}^{+}(H), E \circ B$ should be a positive operator, so when $x, z \geq 0$ and $x z-|y|^{2} \geq 0$, we have $f(x, y, z) \geq 0$. Take $y \in R$, then $f(x, y, z)=x+2 n y$. Thus, when $x, z \geq 0, y \in R$ and $x z-y^{2} \geq 0$, $f(x, y, z)=x+2 n y \geq 0$. If $n \neq 0$, take $y=-\frac{1}{n}, x=1, z=\frac{1}{n^{2}}$, then we have $f<0$, this is a contradiction and so $n=0$. Similarly, if $m \neq 0$, take $y=-\frac{i}{m}, x=1, z=\frac{1}{m^{2}}$, we will get $f<0$, this is also a contradiction and so $m=0$. Thus, we have $f(x, y, z)=x$. Moreover, note that $E \circ((I-E) \circ B)=(E \circ(I-E)) \circ B=0 \circ B=0=((I-E) \circ E)) \circ B=$ $(I-E) \circ(E \circ B)$, as above, we may prove that $\left((I-E) \circ(E \circ B)\left(e_{1}\right),(I-E) \circ(E \circ B)\left(e_{2}\right)\right)=$ $\left(e_{1}, e_{2}\right)\left(\begin{array}{cc}0 & 0 \\ 0 & h(x, y, z)\end{array}\right)=\left(e_{1}, e_{2}\right)\left(\begin{array}{ll}0 & 0 \\ 0 & 0\end{array}\right)$, thus $h(x, y, z)=0$. For each $y \in \mathbb{C}$, take $x=1, z=|y|^{2}$, then $B$ is a positive operator, so $E \circ B$ is also a positive operator, thus we have $f h-|g|^{2} \geq 0$. It follows from $h=0$ that $g=0$, so $E \circ B=X=E B E$.

Corollary 3.2. Let $E \in \mathcal{P}(H), a \in[0,1]$ and $A=a E$. Then for each $B \in \mathcal{E}(H)$,

$$
A \circ B=(a E) \circ B=a(E \circ B)=a(E B E)=a^{\frac{1}{2}} E B a^{\frac{1}{2}} E=A^{\frac{1}{2}} B A^{\frac{1}{2}} .
$$

Now, we prove the following important result: 
Theorem 3.2. Let $H$ be a complex Hilbert space with $\operatorname{dim}(H)=2, A, B \in \mathcal{E}(H)$. If $\left\{e_{1}, e_{2}\right\}$ is a normal basis of $H$ such that $\left(A\left(e_{1}\right), A\left(e_{2}\right)\right)=\left(e_{1}, e_{2}\right)\left(\begin{array}{cc}a^{2} & 0 \\ 0 & b^{2}\end{array}\right)$ and $\left(B\left(e_{1}\right), B\left(e_{2}\right)\right)=\left(e_{1}, e_{2}\right)\left(\begin{array}{ll}x & y \\ \bar{y} & z\end{array}\right)$, then there exists a $\theta \in \mathbb{R}$ such that

$$
\left(A \circ B\left(e_{1}\right), A \circ B\left(e_{2}\right)\right)=\left(e_{1}, e_{2}\right)\left(\begin{array}{cc}
a^{2} x & a b e^{i \theta} y \\
a b e^{-i \theta} \bar{y} & b^{2} z
\end{array}\right) .
$$

Proof. Let $\left\{e_{1}, e_{2}\right\}$ be a normal basis of $H$ such that $\left(A\left(e_{1}\right), A\left(e_{2}\right)\right)=\left(e_{1}, e_{2}\right)\left(\begin{array}{cc}a^{2} & 0 \\ 0 & b^{2}\end{array}\right)$ and $\left(B\left(e_{1}\right), B\left(e_{2}\right)\right)=\left(e_{1}, e_{2}\right)\left(\begin{array}{cc}x & y \\ \bar{y} & z\end{array}\right)$, where $0 \leq a, b \leq 1,0 \leq x, 0 \leq z, 0 \leq x z-|y|^{2}$. Now we define a linear operator $E$ on $H$ such that $\left(E\left(e_{1}\right), E\left(e_{2}\right)\right)=\left(e_{1}, e_{2}\right)\left(\begin{array}{ll}1 & 0 \\ 0 & 0\end{array}\right)$, then $E \in \mathcal{P}(H)$. By Corollary 3.2, we can suppose $a, b \in(0,1]$ and $a \neq b$. Thus, $A=a^{2} E+b^{2}(I-E)$. Denote $\left(A \circ B\left(e_{1}\right), A \circ B\left(e_{2}\right)\right)=\left(e_{1}, e_{2}\right)\left(\begin{array}{ll}f(x, y, z) & g(x, y, z) \\ g(x, y, z) & h(x, y, z)\end{array}\right)$, where $f, g, h$ are real linear functions with respect to $(x, y, z) \in \mathbb{R} \times \mathbb{C} \times \mathbb{R}$ and $f, h$ take values in $\mathbb{R}$. Since $E \circ(A \circ B)=(E \circ A) \circ B)=\left(E \circ\left(a^{2} E+b^{2}(I-E)\right)\right) \circ B=a^{2}(E \circ B)$, we have $f(x, y, z)=a^{2} x$. Similarly, we have also $h(x, y, z)=b^{2} z$. Moreover, since $E|E, E|(I-E)$, by (S5), we have $E \mid A$, so by (S4), we have $(I-E) \mid A$, thus, $A \circ(x E)=$ $x a^{2} E, A \circ z(I-E)=z b^{2}(I-E)$, this showed that $g$ is independent of $x$ and $z$, so $g(x, y, z)=\alpha y$, where $\alpha \in C$. On the other hand, if $B \in \mathcal{S}(H)$ is a positive operator, then $A \circ B$ is also a positive operator, so for each positive number $x$ and $z$, and each complex number $y$, when $x z-|y|^{2} \geq 0$, we have $a^{2} b^{2} x z-|\alpha y|^{2} \geq 0$. Let $x=1, z=|y|^{2}$. Then we get that

$$
a^{2} b^{2}-|\alpha|^{2} \geq 0
$$

Let $B, C$ be two positive operators. We show that if both $B \leq C$ and $C \leq B$ are not true, then both $A \circ B \leq A \circ C$ and $A \circ C \leq A \circ B$ are also not true. In fact, let $D=b^{2} E+a^{2}(I-E)$. Then $A \mid b^{2} E+a^{2}(I-E)=D$ and $A \circ D=A \circ\left(b^{2} E+\right.$ $\left.a^{2}(I-E)\right)=a^{2} b^{2} I$. So if $A \circ B \leq A \circ C$, then $D \circ(A \circ B) \leq D \circ(A \circ C)$. But $D \circ(A \circ B)=(D \circ A) \circ B=a^{2} b^{2} I \circ B=a^{2} b^{2} B \leq D \circ(A \circ C)=a^{2} b^{2} C$, thus we will have $B \leq C$, this is a contradiction. So $A \circ B \leq A \circ C$ is not true. Similarly, we have 
$A \circ C \leq A \circ B$ is also not true.

Let $y \in \mathbb{C}, y \neq 0, \epsilon$ be a positive number satisfy that $a^{2}|y|-\epsilon>0$. If we define $\left(B\left(e_{1}\right), B\left(e_{2}\right)\right)=\left(e_{1}, e_{2}\right)\left(\begin{array}{cc}|y| & y \\ \bar{y} & |y|\end{array}\right)$ and $\left(C\left(e_{1}, C\left(e_{2}\right)\right)=\left(e_{1}, e_{2}\right)\left(\begin{array}{cc}\epsilon & 0 \\ 0 & 0\end{array}\right)\right.$, then $B, C \in \mathcal{E}(H), B \leq C$ and $C \leq B$ are both not true. Thus we have both $A \circ B \leq A \circ C$ and $A \circ B \leq A \circ C$ are also not true, i.e., the self-adjoint operator $A \circ B-A \circ C$ is not positive operator. Note that $\left((A \circ B-A \circ C)\left(e_{1}\right),(A \circ B-A \circ C)\left(e_{2}\right)\right)=\left(e_{1}, e_{2}\right)\left(\begin{array}{cc}a^{2}|y|-\epsilon & \alpha y \\ \overline{\alpha y} & b^{2}|y|\end{array}\right)$, and $a^{2}|y|-\epsilon>0, b^{2}|y|>0$, so we have $b^{2}\left(a^{2}|y|-\epsilon\right)|y|-|\alpha y|^{2}<0$. Let $\epsilon \rightarrow 0$, we get that $|\alpha y|^{2} \geq b^{2} a^{2}|y|^{2}$. Thus, we have

$$
|\alpha|^{2} \geq b^{2} a^{2}
$$

It follows from (1) and (2) that $|\alpha|^{2}=a^{2} b^{2}$. So $|\alpha|=a b$ and $\alpha=a b e^{i \theta}$.

\section{A New Sequential Product on $\mathcal{E}(H)$}

Theorem 3.2 motivated us to construct the new sequential product on $\mathcal{E}(H)$. First, we need the following:

For each $A \in \mathcal{E}(H)$, denote $R(A)=\{A x, x \in H\}, N(A)=\{x, x \in H, A x=0\}, P_{0}$ and $P_{1}$ be the orthogonal projections on $\overline{R(A)}$ and $N(A)$, respectively. It follows from $A \in \mathcal{E}(H)$ that $N(A)=N\left(A^{1 / 2}\right)$, so $R(A)=R\left(A^{1 / 2}\right)$. Moreover, $P_{0}(H) \perp P_{1}(H)$ and $H=P_{0}(H) \oplus P_{1}(H)([10])$.

Denote $f_{z}(u)$ be the complex-valued Borel function defined on $[0,1]$, where $f_{z}(u)=$ $\exp z(\ln u)$ if $u \in(0,1]$ and $f_{z}(0)=0$. Now, we define

$$
A^{i}=f_{i}(A), A^{-i}=f_{-i}(A)
$$

It is easily to show that $\left\|A^{i}\right\| \leq 1,\left\|A^{-i}\right\| \leq 1$ and

$$
\left(A^{i}\right)^{*}=A^{-i}, A^{i} A^{-i}=A^{-i} A^{i}=P_{0} .
$$

Theorem 4.1. Let $H$ be a complex Hilbert space and $A, B \in \mathcal{E}(H)$. If we define $A \circ B=A^{1 / 2} A^{i} B A^{-i} A^{1 / 2}$, then $\circ$ satisfies the conditions (S1)-(S3).

Proof. If $A, B \in \mathcal{E}(H)$, note that $\left\|A^{i}\right\| \leq 1$ and $\left\|A^{-i}\right\| \leq 1$, we have

$$
\|A \circ B\|=\left\|A^{1 / 2} A^{i} B A^{-i} A^{1 / 2}\right\| \leq\left\|A^{1 / 2}\right\|\left\|A^{i}\right\|\|B\|\left\|A^{-i}\right\|\left\|A^{1 / 2}\right\| \leq 1
$$


and

$$
<A^{1 / 2} A^{i} B A^{-i} A^{1 / 2} x, x>=\left\|B^{1 / 2} A^{-i} A^{1 / 2} x\right\| \geq 0
$$

for all $x \in H$, so $A \circ B=A^{1 / 2} A^{i} B A^{-i} A^{1 / 2}$ is a binary operation on $\mathcal{E}(H)$. Moreover, it is clear that the map $B \rightarrow A \circ B$ is additive for each $A \in \mathcal{E}(H)$, so the operation $\circ$ satisfies (S1).

It follows from $I \circ A=I^{1 / 2} I^{i} A I^{-i} I^{1 / 2}=A$ that $\circ$ satisfies (S2).

If $A \circ B=A^{1 / 2} A^{i} B A^{-i} A^{1 / 2}=0$, now, we represent $A$ and $B$ on $H=P_{0}(H) \oplus P_{1}(H)$ by

$$
\begin{aligned}
\left(\begin{array}{cc}
A_{1} & 0 \\
0 & 0
\end{array}\right) \text { and }\left(\begin{array}{cc}
B_{1} & B_{2} \\
B_{3} & B_{4}
\end{array}\right) \text {, then } \\
\qquad A \circ B=\left(\begin{array}{cc}
A_{1}^{1 / 2} A_{1}^{i} B_{1} A_{1}^{-i} A_{1}^{1 / 2} & 0 \\
0 & 0
\end{array}\right)=0,
\end{aligned}
$$

so we have $A_{1}^{1 / 2} A_{1}^{i} B_{1} A_{1}^{-i} A_{1}^{1 / 2}=0$ on $P_{0}(H)$, i.e., $\left(A_{1}^{1 / 2} A_{1}^{i} B_{1} A_{1}^{-i} A_{1}^{1 / 2} x, x\right)=0$ for each $x \in P_{0}(H)$. Note that $R(A)=R\left(A^{1 / 2}\right)$ and $A^{i}$ is a unitary operator on $P_{0}(H)$, so $R\left(A^{1 / 2}\right)$ is dense in $P_{0}(H)$, thus for each $y \in P_{0}(H)$, there is a sequence $\left\{z_{n}\right\} \subseteq R\left(A^{1 / 2}\right)$ such that $z_{n} \rightarrow A^{i} y$, so there is a sequence $\left\{x_{n}\right\} \subseteq H$ such that $A^{1 / 2} x_{n}=z_{n} \rightarrow A^{i} y$. Let $x_{n}=y_{n}+u_{n}$, where $y_{n} \in P_{0}(H), u_{n} \in P_{1}(H)$. Then $A^{1 / 2} x_{n}=A^{1 / 2} y_{n}$. Thus, there is a sequence $\left\{y_{n}\right\}$ in $P_{0}(H)$ such that $A^{1 / 2} y_{n}=z_{n} \rightarrow A^{i} y$. Note that $A^{i}$ is a unitary operator on $P_{0}(H)$, so we have $A^{-i} A^{1 / 2} y_{n} \rightarrow y$. But,

$$
\left\|B_{1}^{1 / 2} A_{1}^{-i} A_{1}^{1 / 2} y_{n}\right\|=\left(A_{1}^{1 / 2} A_{1}^{i} B_{1} A_{1}^{-i} A_{1}^{1 / 2} y_{n}, y_{n}\right)=0
$$

so $B_{1}^{1 / 2} y=0$ for each $y \in P_{0}(H)$, that is, $B_{1}^{1 / 2}=0$. Since $B \in \mathcal{E}(H)$, so $B_{2}=0, B_{3}=0$, thus we have $B=\left(\begin{array}{cc}0 & 0 \\ 0 & B_{4}\end{array}\right)$, so $B \circ A=B^{1 / 2} B^{i} A B^{-i} B^{1 / 2}=0=A \circ B$. This showed that $\circ$ satisfies (S3).

Theorem 4.2. Let $H$ be a complex Hilbert space with $\operatorname{dim}(H)<\infty, A, B \in \mathcal{E}(H)$. If we define $A \circ B=A^{1 / 2} A^{i} B A^{-i} A^{1 / 2}$, then $A \circ B=A^{1 / 2} A^{i} B A^{-i} A^{1 / 2}=B \circ A=$ $B^{1 / 2} B^{i} A B^{-i} B^{1 / 2}$ if and only if $A B=B A$.

Proof. Firstly, it is obvious that if $A B=B A$, then $A \circ B=A^{1 / 2} A^{i} B A^{-i} A^{1 / 2}=B \circ A=$ $B^{1 / 2} B^{i} A B^{-i} B^{1 / 2}$. Now, if $A \circ B=A^{1 / 2} A^{i} B A^{-i} A^{1 / 2}=B \circ A=B^{1 / 2} B^{i} A B^{-i} B^{1 / 2}$, we show that $A B=B A$. Note that $A \in \mathcal{E}(H)$ and $\operatorname{dim}(H)<\infty$, so $A$ has the form $\sum_{i=1}^{n} a_{i} E_{i}$, where $\sum_{k=1}^{n} E_{k}=I, a_{k} \geq 0, E_{k} \in \mathcal{P}(H), a_{k} \neq a_{l}, E_{k} E_{l}=0$ for all $k, l=$ $1,2, \cdots, n, k \neq l$. Without losing generality, we suppose that $0 \leq a_{1}<\cdots<a_{n}$, 
then $0 \leq\left|a_{1}^{1 / 2} f_{i}\left(a_{1}\right)\right|<\cdots<\left|a_{n}^{1 / 2} f_{i}\left(a_{n}\right)\right|$ since $a_{k}^{1 / 2}=\left|a_{k}^{1 / 2} f_{i}\left(a_{k}\right)\right|$. It follows from the operator theory that $A^{1 / 2}=\sum_{k=1}^{n} a_{k}^{1 / 2} E_{k}$ and $f_{i}(A)=A^{i}=\sum_{k=1}^{n} f_{i}\left(a_{k}\right) E_{k}, f_{-i}(A)=A^{-i}=$ $\sum_{k=1}^{n} f_{-i}\left(a_{k}\right) E_{k}([10])$. Note that $A^{1 / 2} A^{i} B A^{-i} A^{1 / 2}=B^{1 / 2} B^{i} A B^{-i} B^{1 / 2}$, so for each $x \in H$, $\left(A^{1 / 2} A^{i} B A^{-i} A^{1 / 2} x, x\right)=\left(B^{1 / 2} B^{i} A B^{-i} B^{1 / 2} x, x\right)$, thus we have

$$
\left\|B^{1 / 2} A^{-i} A^{1 / 2} x\right\|=\left\|A^{1 / 2} B^{-i} B^{1 / 2} x\right\| .
$$

Take $x \in E_{n}(H)$, then $A^{1 / 2} A^{-i} x=A^{-i} A^{1 / 2} x=a_{n}^{1 / 2} f_{-i}\left(a_{n}\right) x$, note that $\left|a_{n} f_{-i}\left(a_{n}\right)\right|=$ $\left|a_{n} f_{i}\left(a_{n}\right)\right|=\left|a_{n}\right|, \overline{R(B)}=\overline{R\left(B^{1 / 2}\right)}$ and $B^{-i}$ is a unitary operator on $\overline{R(B)}$ and $B^{-i} B^{1 / 2}=$ $B^{1 / 2} B^{-i}$, we have

$$
\begin{gathered}
\left\|A^{1 / 2} B^{1 / 2} B^{-i} x\right\|^{2}=\left\|\sum_{k=1}^{n} a_{k}^{1 / 2} E_{k} B^{1 / 2} B^{-i} x\right\|^{2}= \\
\sum_{k=1}^{n} a_{k}\left\|E_{k} B^{1 / 2} B^{-i} x\right\|^{2} \leq \sum_{k=1}^{n} a_{n}\left\|E_{k} B^{1 / 2} B^{-i} x\right\|^{2}= \\
a_{n}\left\|B^{1 / 2} B^{-i} x\right\|^{2}=\left\|a_{n}^{1 / 2} B^{-i} B^{1 / 2} x\right\|^{2}= \\
\left\|a_{n}^{1 / 2} B^{1 / 2} x\right\|^{2}=\left\|B^{1 / 2} A^{1 / 2} A^{-i} x\right\|^{2} .
\end{gathered}
$$

Thus, it follows from equation (3), $B^{-i} B^{1 / 2}=B^{1 / 2} B^{-i}, A^{-i} A^{1 / 2}=A^{1 / 2} A^{-i}$ and $0 \leq$ $a_{1}<\cdots<a_{n}$ that for each $k<n$, we have $E_{k} B^{1 / 2} B^{-i} x=0$, so $B^{1 / 2} B^{-i} x \in E_{n}(H)$. Thus we have $E_{n} B^{1 / 2} B^{-i} E_{n}=B^{1 / 2-i} E_{n}$. This showed that $B^{1 / 2} B^{-i}$ has the matrix form $\left(\begin{array}{cc}C & D \\ 0 & K\end{array}\right)$ on $H=E_{n}(H) \oplus\left(I-E_{n}\right)(H)$, where $C \in \mathcal{B}\left(E_{n}(H), E_{n}(H)\right), D \in$ $\mathcal{B}\left(\left(I-E_{n}\right)(H), E_{n}(H)\right), K \in \mathcal{B}\left(\left(I-E_{n}\right)(H),\left(I-E_{n}\right)(H)\right)$. Note that $B \in \mathcal{E}(H), B$ has the form $\sum_{k=1}^{m} b_{k} F_{k}$, and $B^{1 / 2} B^{-i}=\sum_{k=1}^{m} b^{1 / 2} f_{-i}\left(b_{k}\right) F_{k}$, where $\sum_{k=1}^{m} F_{k}=I, b_{k} \geq 0$, $F_{k} \in \mathcal{P}(H), b_{k} \neq b_{l}, F_{k} F_{l}=0$ for all $k, l=1,2, \cdots, m, k \neq l$. Now we define a polynomial

$$
G_{k}(z)=\prod_{j \neq k}\left(z-b_{j}^{1 / 2} f_{-i}\left(b_{j}\right)\right) / \prod_{j \neq k}\left(b_{k}^{1 / 2} f_{-i}\left(b_{j}\right)-b_{j}^{1 / 2} f_{-i}\left(b_{j}\right)\right)
$$

on $\mathbb{C}$. It is easily to show that for each $1 \leq k \leq m, G_{k}\left(B^{1 / 2} B^{-i}\right)=F_{k}$. Note that $B^{1 / 2} B^{-i}$ has the up-triangulate form, so $G_{k}\left(B^{1 / 2} B^{-i}\right)$ has also the up-triangulate form. But $F_{k}$ is a self-adjoint operator, so $F_{k}$ has the diagonal matrix form on $E_{n}(H) \oplus\left(I-E_{n}\right)(H)$. This implies that $F_{k}$ commutes with $E_{n}$ for each $k$, so $B$ commutes with $E_{n}$. Denote $A_{0}=A-a_{n} E_{n}$, then we still have $A_{0} \circ B=B \circ A_{0}$ as discussed before, thus we get that 
$B$ commutes with $E_{n-1}$. Continuously, we will have that $B$ commutes with all $E_{k}$ and so with $\mathrm{A}$. In this case we have $A \circ B=A B$.

Our main result is:

Theorem 4.3. Let $H$ be a complex Hilbert space with $\operatorname{dim}(H)<\infty$ and $A, B \in \mathcal{E}(H)$. If we define $A \circ B=A^{1 / 2} A^{i} B A^{-i} A^{1 / 2}$, then $\circ$ is a sequential product on $\mathcal{E}(H)$.

Proof. By Theorem 4.1, we only need to prove that $\circ$ satisfies (S4) and (S5). In fact, if $A \mid B$, i.e., $A \circ B=A^{1 / 2} A^{i} B A^{-i} A^{1 / 2}=B \circ A=B^{1 / 2} B^{i} A B^{-i} B^{1 / 2}$, then it follows from Theorem 4.2 that $A$ commutes with $B$ and of course $I-B$, so $A \mid I-B$. If $C \in \mathcal{E}(H)$, we have

$$
\begin{gathered}
A \circ(B \circ C)=A^{\frac{1}{2}} A^{i} B^{\frac{1}{2}} B^{i} C B^{-i} B^{\frac{1}{2}} A^{-i} A^{\frac{1}{2}} \\
=A^{\frac{1}{2}} B^{\frac{1}{2}} A^{i} B^{i} C A^{-i} B^{-i} A^{\frac{1}{2}} B^{\frac{1}{2}} \\
=(A B)^{\frac{1}{2}}(A B)^{i} C(A B)^{-i}(A B)^{\frac{1}{2}} \\
=(A B) \circ C=(A \circ B) \circ C .
\end{gathered}
$$

So (S4) is satisfied.

Moreover, if $C \mid B$ and $C \mid A$, then $C(A B)=A C B=(A B) C, C(A \oplus B)=(B+A) C$, so it is easily to prove that $C(A \circ B)=(A \circ B) C$, thus, by Theorem 4.2 , we have $C \mid A \circ B$ and $C \mid(A \oplus B)$ whenever $A \oplus B$ is defined, this showed that (S5) is hold.

By using Theorem 4.3 we can prove the following corollary:

Corollary 4.4. Let $H$ be a complex Hilbert space with $\operatorname{dim}(H)=2, A, B \in \mathcal{E}(H)$. Take a normal basis $\left\{e_{1}, e_{2}\right\}$ of $H$ such that $\left(A\left(e_{1}\right), A\left(e_{2}\right)\right)=\left(e_{1}, e_{2}\right)\left(\begin{array}{cc}a^{2} & 0 \\ 0 & b^{2}\end{array}\right)$ and $\left(B\left(e_{1}\right), B\left(e_{2}\right)\right)=\left(e_{1}, e_{2}\right)\left(\begin{array}{ll}x & y \\ \bar{y} & z\end{array}\right)$. If when $a, b>0$, define

$$
\left((A \circ B)\left(e_{1}\right),(A \circ B)\left(e_{2}\right)\right)=\left(e_{1}, e_{2}\right)\left(\begin{array}{cc}
a^{2} x & a b e^{i \theta} y \\
a b e^{-i \theta} \bar{y} & b^{2} z
\end{array}\right),
$$

where $\theta=\ln a^{2}-\ln b^{2} ;$ when $a>0, b=0$, define

$$
\left((A \circ B)\left(e_{1}\right),(A \circ B)\left(e_{2}\right)\right)=\left(e_{1}, e_{2}\right)\left(\begin{array}{cc}
a^{2} x & 0 \\
0 & 0
\end{array}\right),
$$


when $a=0, b>0$, define

$$
\left((A \circ B)\left(e_{1}\right),(A \circ B)\left(e_{2}\right)\right)=\left(e_{1}, e_{2}\right)\left(\begin{array}{cc}
0 & 0 \\
0 & b^{2} z
\end{array}\right),
$$

then $\circ$ is a sequential product of $\mathcal{E}(H)$.

Remark 1. In conclusion, we construct a new sequential product $A \circ B=A^{\frac{1}{2}} A^{i} B A^{-i} A^{\frac{1}{2}}$ on $\varepsilon(H)$ with $\operatorname{dim}(H)<\infty$, which is different from the generalized Lüders form $A^{\frac{1}{2}} B A^{\frac{1}{2}}$. In this proof we can also get a more general one $A \circ B=A^{\frac{1}{2}} A^{t i} B A^{-t i} A^{\frac{1}{2}}$ for $t \in R$. It indicates that with the measurement rule (S1)-(S5), there can be a time parameter $t$ to describe the phase change. In particular, if $\operatorname{dim}(H)=2, A \in \mathcal{E}(H)$ and $\left\{e_{1}, e_{2}\right\}$ is a normal basis of $H$ such that $\left(A\left(e_{1}\right), A\left(e_{2}\right)\right)=\left(e_{1}, e_{2}\right)\left(\begin{array}{cc}a^{2} & 0 \\ 0 & b^{2}\end{array}\right)$, then when $a>0, b>0$ and $a \neq b$, Corollary 4.4 showed that $\theta=\left(\ln a^{2}-\ln b^{2}\right) t$ can be used to describe the phase-changed phenomena of quantum effect $A \circ B$. As the proof showed, it is the only form that the sequential product can be. This is much more important in physics.

Remark 2. As we knew, in the quantum computation and quantum information theory, if $\left(A_{i}\right)_{i=1}^{n} \subseteq \mathcal{B}(H)$ satisfying $\sum_{i=1}^{n} A_{i} A_{i}^{*}=I$, then the operators $\left(A_{i}\right)_{i=1}^{n}$ are called the operational elements of the quantum operation $U: \mathcal{T}(H) \rightarrow \mathcal{T}(H)$ defined by

$$
U(\rho)=\sum_{i=1}^{n} A_{i} \rho A_{i}^{*}
$$

where $\mathcal{T}(H)$ is the set of trace class operators. Any trace preserving, normal, completely positive map has the above form. This is very important in describing dynamics, measurements, quantum channels, quantum interactions, and quantum error, correcting codes, etc. If $\left(A_{i}\right)_{i=1}^{n}$ is a set of quantum effects with $\sum_{i=1}^{n} A_{i}=I$, then the transformation $U^{\prime}(\rho)=\sum_{j=1}^{n} A_{j}^{\frac{1}{2}} A_{j}^{t i} \rho A_{j}^{-t i} A_{j}^{\frac{1}{2}}$ is a well defined quantum operation since $\sum_{j=1}^{n} A_{j}^{\frac{1}{2}} A_{j}^{t i} A_{j}^{-t i} A_{j}^{\frac{1}{2}}=\sum_{i=1}^{n} A_{i}=I$. So this new sequential product yields a natural and interesting quantum operation.

Remark 3. Theorem 4.3 indicates that the conditions (S1)-(S5) of sequential product of $\mathcal{E}(H)$ are not sufficient to characterize the generalized Lüders form $A^{\frac{1}{2}} B A^{\frac{1}{2}}$ of $A$ and $B$. Recently, Professor Gudder presented a characterization of the sequential product of $\mathcal{E}(H)$ is the generalized Lüders form $([11])$. 
Acknowledgement. The authors wish to express their thanks to the referees for their valuable comments and suggestions. In particular, their comments motivated the authors to prove Theorem 4.3 for any finite dimensional Hilbert spaces. This project is supported by Natural Science Foundations of China (10771191 and 10471124).

\section{References}

[1] Ludwig G 1983 Foundations of Quantum Mechanics (I-II) (Springer, New York)

[2] Ludwig G 1986 An Axiomatic Basis for Quantum Mechanics (II) (Springer, New York)

[3] Kraus K 1983 Effects and Operations (Springer-Verlag, Beilin)

[4] Davies E B 1976 Quantum Theory of Open Systems (Academic Press, London)

[5] Busch P, Grabowski M and Lahti P J 1999 Operational Quantum Physics (SpringerVerlag, Beijing Word Publishing Corporation)

[6] Gudder S, Nagy G 2001 J. Math. Phys. 425212

[7] Gudder S, Greechie R 2002 Rep Math. Phys. 4987

[8] Gheondea A, Gudder S 2004 Proc. Am. Math. Soc. 132503

[9] Gudder S 2005 Inter. J. Theory. Physi. 442199

[10] Kadison R V, Ringrose J R 1983 Fundamentals of the Theory of Operator algebra (Springer, New York)

[11] Gudder S, Latremoliere F 2008 J. Math. Phys. 49052106 